\title{
Cardiothoracic
}

Transplantation

\section{Simvastatin attenuates cardiac isograft ischemia-reperfusion injury by down-regulating CC chemokine receptor-2 expression}

Rong Yin, MD, Jiaquan Zhu, MD, Zhongqiu Wang, MD, Hairong Huang, MD, Jianjun Oian, MD, Zhongdong Li, MD, and Hua Jing, MD

From the Department of Cardiothoracic Surgery, Jinling Hospital, Clinical Medicine School of Nanjing University, Nanjing, China.

Received for publication Dec 1, 2006; revisions received April 19, 2007; accepted for publication May 2, 2007.

Reprint requests: Hua Jing, MD, Department of Cardiothoracic Surgery, Jinling Hospital, Clinical Medicine School of Nanjing University, 305 East Zhongshan Road, Nanjing 210002, China (E-mail: Jing hua_1@yahoo.com.cn).

J Thorac Cardiovasc Surg 2007;134:780-8

$0022-5223 / \$ 32.00$

Copyright () 2007 by The American Association for Thoracic Surgery

doi:10.1016/j.jtcvs.2007.05.001
Objective: Accumulating evidence reveals that statins possess direct anti-inflammatory properties through inhibition of proinflammatory cytokine and chemokine secretion in addition to their antioxidant effects, which may contribute to amelioration of ischemia-reperfusion injury. This study tested the hypothesis that perioperative treatment of simvastatin suppresses the cardiac isograft ischemia-reperfusion injury by down-regulation of $\mathrm{CC}$ chemokine receptor- 2 expression in an inbred rat model of cardiac transplantation.

Methods: Donor hearts from Lewis rats were heterotopically transplanted to Lewis rat recipients. Recipients were orally treated with simvastatin $(1 \mathrm{mg} / \mathrm{kg})$ or vehicle every morning 3 days before the surgery until the harvest day. Rats were killed at 6 hours and at 1, 3, and 7 days after transplantation. Injury was assessed by infarct size measurement, histologic and immunohistochemical examination, and intragraft myeloperoxidase activity assay. Monocyte chemoattractant protein-1 levels in serum and graft were analyzed by enzyme-linked immunosorbent assay, and intragraft $\mathrm{CC}$ chemokine receptor- 2 expression was measured by quantitative real-time polymerase chain reaction.

Results: The infarct size and macrophage infiltration were all significantly reduced in the simvastatin-treated group compared with those of the control group at 1 day after transplantation. Neutrophil accumulation was significantly suppressed until 3 days after transplantation, whereas myeloperoxidase activity had been significantly diminished at 1 day after transplantation. Both monocyte chemoattractant protein-1 concentrations in serum and graft were remarkably decreased at 6 hours after transplantation. Intragraft CC chemokine receptor-2 expression was also downregulated at 1 day and 3 days after transplantation.

Conclusions: Perioperative treatment of simvastatin could suppress the isograft ischemia-reperfusion injury through retarding intragraft monocyte chemoattractant protein- 1 accumulation and $\mathrm{CC}$ chemokine receptor- 2 expression.

$\mathrm{I}$ schemia-reperfusion injury (IRI) is of paramount importance to organ transplantation and is a major determinant of early graft dysfunction. ${ }^{1}$ If overwhelmed, IRI might exert continuous and deleterious effects on allografts. During IRI, secretion of intragraft proinflammatory cytokines, chemokines, and 


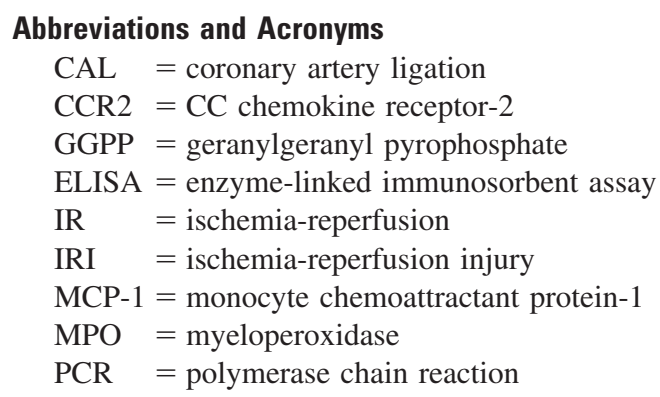

adhesion molecules is increased, and such mediators could promote adhesion and migration of alloreactive $\mathrm{T}$ lymphocytes, ultimately triggering the acute rejection process. ${ }^{2}$ In addition, several earlier studies have also demonstrated that the extent or duration of IRI is positively correlated with the degree of cardiac allograft vasculopathy and allograft longterm survival. ${ }^{3,4}$ Therefore, maximal reduction of IRI should markedly decrease the rates of acute and chronic rejection and prolong the survival of grafts.

Leukocyte recruitment is essential for the development of IRI and involves graft endothelia up-regulation of adhesion molecules, selectins, and especially chemokines. ${ }^{1}$ Over the past years, chemokines and their receptors have become the subject of intensive investigations. Chemokine expression is a prominent feature of the IRI inflammatory response and may play an important role in leukocyte recruitment. ${ }^{5,6}$ One of the best-studied CC chemokines, monocyte chemoattractant protein-1 (MCP-1), is reported to be the key molecule in terms of chemotaxis and activation of macrophages, with the CC chemokine receptor-2 (CCR2) as its major receptor. MCP-1 up-regulation has been observed in experimental myocardial infarction of canines, rats, and mice. ${ }^{7}$ Growing evidence indicates that blockade of MCP-1/ CCR2 signaling could markedly retard macrophage infiltration and activation in IRI. For example, recent studies using gene knockout mice demonstrated that both CCR2 and MCP-1 deficiencies alleviate myocardial or renal IRI. ${ }^{6,8,9}$ Moreover, the MCP-1/CCR2 axis displayed the role of contributing neutrophil recruitment through the cooperativity of monocyte in recent investigations, although neutrophils do not express CCR2 or respond to MCP-1 directly. ${ }^{10}$

In the past decade, more and more studies have revealed a variety of cardiovascular benefits of 3-hydroxyl-3methylglutaryl coenzyme A reductase inhibitors (statins) beyond their well-known cholesterol-lowering property. ${ }^{11}$ These so-called pleiotropic effects encompass antiinflammation, correction of endothelial dysfunction, increase in nitric oxide bioavailability, antioxidation, and stabilization of atherosclerotic plaques. ${ }^{12}$ Several mechanisms have been proposed for statin-elicited beneficial effects, including the prevention of mevalonate formation and subsequently the synthesis of isoprenoid farnesyl pyrophosphate and geranylgeranyl pyrophosphate (GGPP), which leads to inhibition of the isoprenylation of small guanosine triphosphate-binding proteins, such as Rho or Ras proteins involved in cell differentiation, apoptosis, and inflammatory response. ${ }^{11}$ More important, previous studies demonstrated that statins may significantly attenuate myocardial reperfusion injury using a coronary artery ligation (CAL) model ${ }^{13,14}$ or Langendorff system. ${ }^{15}$ However, it is still not clear whether IRI in cardiac grafts may be ameliorated by perioperative treatment of statins. In addition, recent in vitro studies displayed that simvastatin could down-regulate chemokine receptors CCR1, CCR2, and CCR5 expression. ${ }^{16}$ We therefore hypothesized that perioperative treatment of simvastatin could suppress the cardiac IRI by regulating MCP-1/ CCR2 signaling in an inbred rat model of cardiac transplantation.

\section{Materials and Methods Animals and Groups}

All inbred male rats (200-250 g) were purchased from Vital River Ltd (Beijing, PR China). Lewis rats served as both donors and recipients. All animals received humane care in compliance with The Principles of Laboratory Animal Care, formulated by the National Society of Medical Research, and The Guide for the Care and Use of Laboratory Animals, published by the U.S. National Institutes of Health (Publication No. 85-23, revised 1996). The experimental protocol described in this study was approved by the local institutional ethical committee.

Rats were randomly divided into 3 groups: the control group $(\mathrm{CON})(\mathrm{n}=28)$, simvastatin-treated group (SIM) $(\mathrm{n}=28)$, and sham group $(\mathrm{n}=24)$. In the first 2 groups, recipients were orally administrated vehicle (3 $\mathrm{mL}$ of saline) or simvastatin (Merck Sharp and Dohme, Hangzhou, PR China, $1 \mathrm{mg} / \mathrm{kg}$ ) every morning. The dosage of simvastatin was described previously, ${ }^{17}$ which is equivalent to $75 \mathrm{mg}$ of simvastatin in humans. Drug administration was begun 3 days before surgery and continued through the day of heart harvest. The sham procedure consisted of anesthesia, abdominal sectioning, and closure of the wounds.

\section{Surgical Procedure}

The donor hearts were heterotopically transplanted into the abdomens of recipients as previously described by Ono and Lindsey. ${ }^{18}$ Briefly, animals were anesthetized with an intraperitoneal injection of pentobarbital $(50 \mathrm{mg} / \mathrm{kg})$. The donor was given heparin (1000 $\mathrm{U} / \mathrm{kg}$ ), and cardiac arrest was induced in the donor heart by the injection of ice-cold, high-potassium cardioplegia solution into the aortic root. The procured hearts were quickly cooled in iced heparinized saline. The recipient was not given heparin. The aorta and pulmonary artery of the donor heart were anastomosed end to side to the recipient abdominal aorta and inferior vena cava, respectively. The grafts were consistently cooled during the procedure with iced saline. We controlled the total cold ischemic time of grafts for 45 minutes and the total ischemic time for 50 minutes in each group. Cardiac isograft function was assessed by daily abdominal palpation. 


\section{Specimen Collection}

In each group, rats were sacrificed at 6 hours and at 1, 3, and 7 days after transplantation ( $\mathrm{n}=6$ in each time point). The blood samples were collected at the time of sacrifice for chemokines enzymelinked immunosorbent assay (ELISA) and cholesterol analysis. The grafts were transversely sectioned into 2 portions. The basal part was used for histologic and immunohistochemical examination. The remaining part was used for ELISA, myocardial myeloperoxidase (MPO) activity assay, and chemokine receptor quantitative real-time polymerase chain reaction (PCR) analysis. In addition, in both the control and SIM groups, 4 isografts harvested at 1 day after transplantation were randomly selected for infract size assessment. The specimens of sham group were obtained from in situ hearts.

\section{Infarct Size Assessment}

Infarct size analysis was performed by 2, 3, 5-triphenyl tetrazolium chloride staining (1\% in phosphate buffer, $\mathrm{pH} 7.4$ ) as previously described. ${ }^{15}$ One day after transplantation, the rats were anesthetized again and the abdomen was reopened. The hearts were harvested and frozen at $-20^{\circ} \mathrm{C}$. Once solid, the hearts were sliced from apex to base into 5 to 7 sections and then incubated in $10 \mathrm{~mL}$ of 2, 3, 5-triphenyl tetrazolium chloride at $37^{\circ} \mathrm{C}$ for 60 minutes. Subsequently, the slices were destained for not less than 12 hours in $10 \%$ formalin to increase the definition between viable and nonviable tissue. The slices were then photographed and planimetered using Image $\mathbf{J}(1.37 \mathrm{v}, \mathrm{NIH}$, Bethesda, MD). The area at risk was then assessed as being the total ventricular volume (minus the chamber spaces), and infarct size was expressed as a percentage of the area at risk (infarct size/area at risk \%).

\section{Histologic and Immunohistochemical Examination}

The basal part of graft was placed in $10 \%$ phosphate-buffered formalin for at least 24 hours. At least four 4- $\mu$ m slides of each specimen were stained with Masson's trichrome for evaluation of fibrosis grade, as previously described. ${ }^{19}$ An immunohistochemical study was also done on formalin-fixed, paraffin-embedded sections. The following mouse-anti-rat monoclonal antibodies were used: ED1, a murine immunoglobulin-G1 anti-rat CD68 (macrophages/monocytes) (Serotec, Oxford, UK, MCA341R); and HIS4, a murine immunoglobulin-M anti-rat neutrophil (Serotec, MCA967). Positively stained cells were counted by an investigator in a blind fashion.

\section{Determination of Myeloperoxidase Activity}

MPO activity was determined as an index of neutrophil accumulation in the IR myocardium as described previously. ${ }^{20}$ Myocardial samples were homogenized in a phosphate buffer containing $0.5 \%$ hexadecyltrimethylammonium bromide. Samples were then assayed for the ability to decompose $\mathrm{H}_{2} \mathrm{O}_{2}$ in the presence of O-dianisidine dihydrochloride by the change in absorption at $460 \mathrm{~nm}$ during 1 minute. The tissue MPO activity was expressed in units per gram of wet tissue. One unit of MPO is defined as that quantity of enzyme hydrolyzing $1 \mu \mathrm{mol}$ peroxide at $37^{\circ} \mathrm{C}$.

Determination of Monocyte Chemoattractant Protein-1 in Serum and Isograft Tissue

MCP-1 concentration in serum and isograft tissues was quantified using an ELISA kit specific for the rat chemokine per the manu- facturer's instructions (RapidBio Lab, Calabasas, Calif). Values were expressed as pictograms per milliliter for serum, and picogram per $100 \mathrm{mg}$ of protein for tissue samples.

\section{CCR2 mRNA Real-time Polymerase Chain Reaction Analysis}

Total cellular RNA was isolated from cardiac tissue by using Trizol Reagents (Invitrogen Life Technologies, Carlsbad, Calif) according to the manufacturer's directions. RNA quality was insured by gel visualization and spectrophotometric analysis $\left(\mathrm{OD}_{260 / 280}\right)$. The quantity of RNA was measured using the $\mathrm{OD}_{260}$. RNA was transcribed to cDNA using Moloney murine leukemia virus reverse transcriptase (Promega, Madison, Wis) and oligo dT primers. Quantitative realtime PCR analysis was performed by using the Rotor-Gene 3000 real-time DNA analysis system (Corbett Research, Sydney, Australia), applying the real-time SYBR Green PCR technology. The reaction mixtures contained diluted cDNA, SYBR Green I Nucleic Acid Stain (Invitrogen Life Technologies), and $20 \mu \mathrm{M}$ each gene-specific primer and nuclease-free water to a final volume of $25 \mu \mathrm{L}$. The PCR thermal cycle conditions were as follows: initial step at $9^{\circ} \mathrm{C}$ for 5 minutes, followed by 40 cycles at $94^{\circ} \mathrm{C}$ for 20 seconds, $6^{\circ} \mathrm{C}$ for 20 seconds, and $7^{\circ} \mathrm{C}$ for 30 seconds. Test cDNA results were normalized to $\beta$-actin measured on the same plate. The primer was designed by using the Primer Premier 5.0 program (PREMIER Biosoft International, Palo Alto, Calif).

\section{Measurement of Lipid Profiles in Plasma}

Lipid profiles were measured using an automated analyzer (Hitachi-917, Hitachi Ltd, Tokyo, Japan).

\section{Statistical Analysis}

All results in the text are expressed as mean \pm standard deviation. Data were analyzed using a commercially available statistics software package (Statistical Package for the Social Sciences for Windows v. 13.0, SPSS Inc, Chicago, Ill). Infarct size between paired groups was analyzed by the Student paired $t$ test. Fibrosis grade, ED1, and HIS4-positive cells count between different groups were analyzed by the Mann-Whitney $U$ test. The 1-way analysis of variance was used to compare experimental data of MPO, real-time PCR, and ELISA between multiple groups. Post hoc comparisons were performed using the Tukey test or Dunnett's T3 test.

\section{Results}

\section{Infarct Size Data}

Representative slices of hearts from the control and SIM groups are shown in Figure 1, A. One day after transplantation, simvastatin treatment significantly reduced the infarct size compared with the control group $(26.65 \% \pm$ $2.48 \%$ vs $41.46 \% \pm 7.90 \%, P=.021$, Figure $1, B)$.

\section{Histologic and Immunohistochemical Examination Results}

As shown in Figure 2, simvastatin treatment significantly decreased the fibrosis grade at 7 days after transplantation 

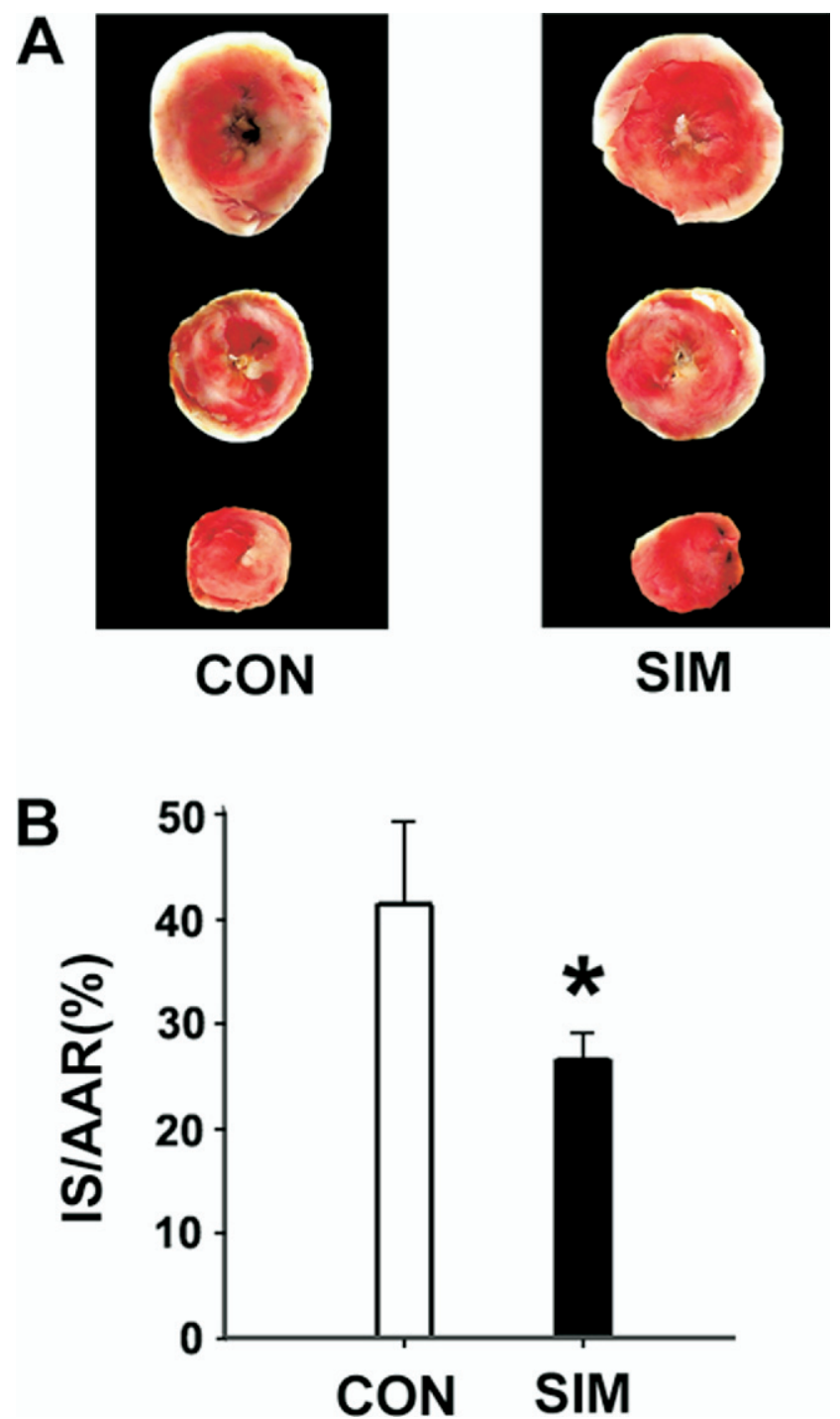

Figure 1. A, Representative slices of hearts from the CON and SIM groups at 1 day after transplantation. Slices were stained with 2, 3, 5-triphenyl tetrazolium chloride. The risk but viable area (red) and the infarct area (white-gray). B, Effects of simvastatin on infarct size. Mean \pm standard deviation, $n=4$ per group. ${ }^{*} P<$ .05 versus control group. $I S$, Infarct size; $A A R$, area at risk.

compared with the control group $(P=.004)$. At both 1 day and 3 days after transplantation, the number of ED1positive cells in the SIM group was markedly reduced compared with the control group $(P=.009$ and $P=.002$, respectively). In addition, although the number of neutrophils was comparable between the control and SIM groups $(P=.394) 1$ day after transplantation, neutrophil infiltration in the SIM group was significantly suppressed compared with the control group $(P=.004) 3$ days after transplantation.
Effects of Simvastatin on Myeloperoxidase Activity In both the control and SIM groups, the MPO activity gradually increased, peaking 1 day after transplantation before gradually decreasing (Figure 3). At 1 day and 3 days after transplantation, the MPO activity in the SIM group was reduced compared with the control group $(P=.01, P=$ .049 , respectively). However, no statistical significance was reached at 6 hours and 7 days after transplantation.

Effects of Simvastatin on Monocyte Chemoattractant Protein-1 Concentration in Serum and Isograft Tissue As shown in Figure 4, A, the serum concentration of MCP-1 in the control group was rapidly elevated to a large extent at 6 hours after transplantation and gradually decreased at subsequent time points compared with the sham group. The SIM group had a similar time course and displayed significant reduction of MCP-1 level at all time points compared with the control group $(P=.009, P=.02, P<.001$, and $P=.001$, respectively). Similarly, the intragraft concentration of MCP-1 in the control group (Figure 4, $B$ ) was rapidly elevated at 6 hours after transplantation before starting to decrease gradually. In contrast, intragraft MCP-1 level in the SIM group was suppressed and showed a delayed peak 1 day after transplantation in comparison with the control group. At the first 3 time points, the intragraft MCP-1 levels were markedly reduced in the SIM group compared with the control group $(P=.001, P=.029$, and $P=.013$, respectively).

\section{Effects of Simvastatin on CC Chemokine Receptor-2 mRNA Expression}

We analyzed intragraft chemokine receptor CCR2 mRNA expression by quantitative real-time PCR (Figure 5). The isografts in control group displayed a significant up-regulation in the expression of CCR2 compared with the sham group, peaking at 6 hours after transplantation. The expression of CCR2 was similar between the SIM and the control groups $(P=.697)$ at the first time point. Subsequently, CCR2 expression in the SIM group was markedly down-regulated compared with the control group at 1,3 , and 7 days after transplantation $(P<.001, P=.003$, and $P=.013$, respectively).

\section{Lipid Profiles of Plasma}

Treatment with simvastatin at our conditions did not significantly alter the plasma total cholesterol level $(61.48 \pm 3.58$ $\mathrm{mg} / \mathrm{dL}$ vs $64.26 \pm 4.39 \mathrm{mg} / \mathrm{dL}$ in the control group, $P=$ .467), indicating that simvastatin-induced attenuation of IRI was independent of its plasma cholesterol-lowering effects.

\section{Discussion}

The major findings of our study indicated that the perioperative administration of simvastatin significantly decreased infarct size and inflammatory cell infiltration of cardiac 


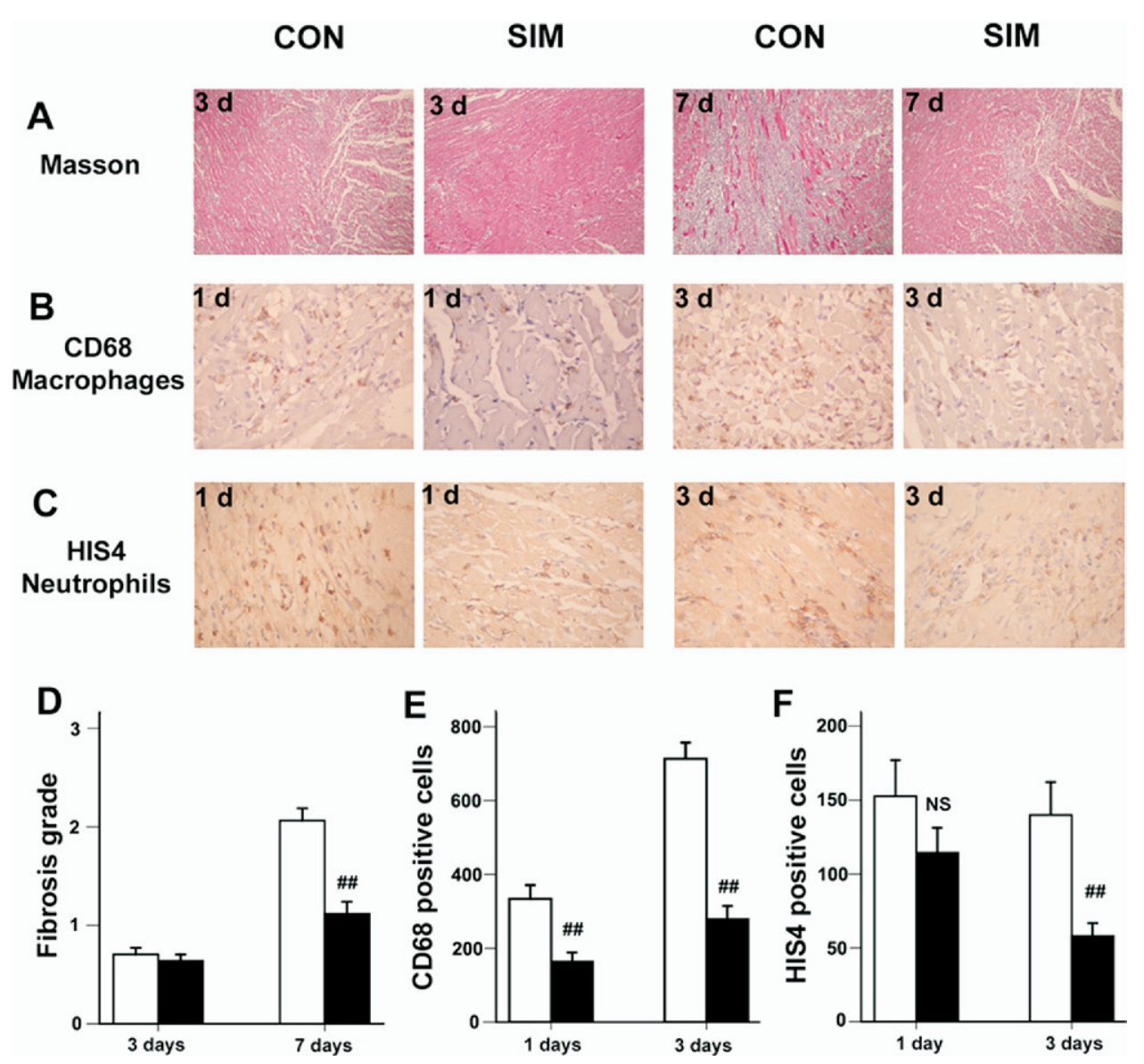

Figure 2. Representative slices of donor hearts from CON and SIM recipients. Simvastatin significantly attenuated cell interstitial fibrosis (A) (Masson, $\times 200$ ), macrophage (B) (CD68 stain $\times 400)$, and neutrophil (C) (HIS4 stain, $\times 400)$ infiltration. Effects of simvastatin treatment on interstitial fibrosis (D), macrophage (E), and neutrophil (F) infiltration at different time points. Mean \pm standard error of the mean $(S E M), n=6$ rats per time point in each group. \#\# $<$ .01 between control (open bars) and simvastatin (filled bars) groups, NS, Not significant.

isografts, resulting in attenuated IRI. Furthermore, simvastatin displayed the effects on reduction of MCP-1 level and down-regulation of CCR2 expression, which may play a role in simvastatin-induced IRI suppression.

Increasing studies have revealed that the treatment of animals with statins attenuates myocardial IRI. ${ }^{13-15,21-26}$ However, most of the studies were performed using the in vitro isolated perfused heart model (Langendorff system) or the in vivo CAL model, which failed to address the issue whether the heart transplantation recipients might benefit from the perioperative statin therapy. Both Langendorff and CAL models have disadvantages for the adequate evaluation of this issue. The CAL model could only simulate regional myocardial ischemia-reperfusion (IR) but not global heart IR during transplantation, whereas the Langendorff system is performed in vitro and does not take the system circulation and many peripheral complications into account, such as inflammatory cell infiltration. Furthermore, the isolated heart rapidly deteriorates and often endures no more than 24 hours. ${ }^{27}$ Therefore, we used this model performed in vivo, which is closer to the clinical transplantation and persists for a longer time. Nonetheless, we cannot completely simulate the clinical environment to obtain hemodynamics data from this model.

Various methods were used by previous investigators with regard to the administration protocol of statin. Most of the studies showed the protective effect of statin-administered agents before the beginning of IR. However, the length of pretreatment time was different. Some investigators applied statins just several hours, 20 minutes, or 3 minutes before ischemia, ${ }^{14,24,28}$ or even at the onset of reperfusion ${ }^{22}$ acutely. Other studies used chronic treatment of statins for 2 days,${ }^{13} 5$ days, ${ }^{20}$ or even 3 weeks ${ }^{25}$ before the procedure. Nevertheless, a recent study of the Langendorff system 


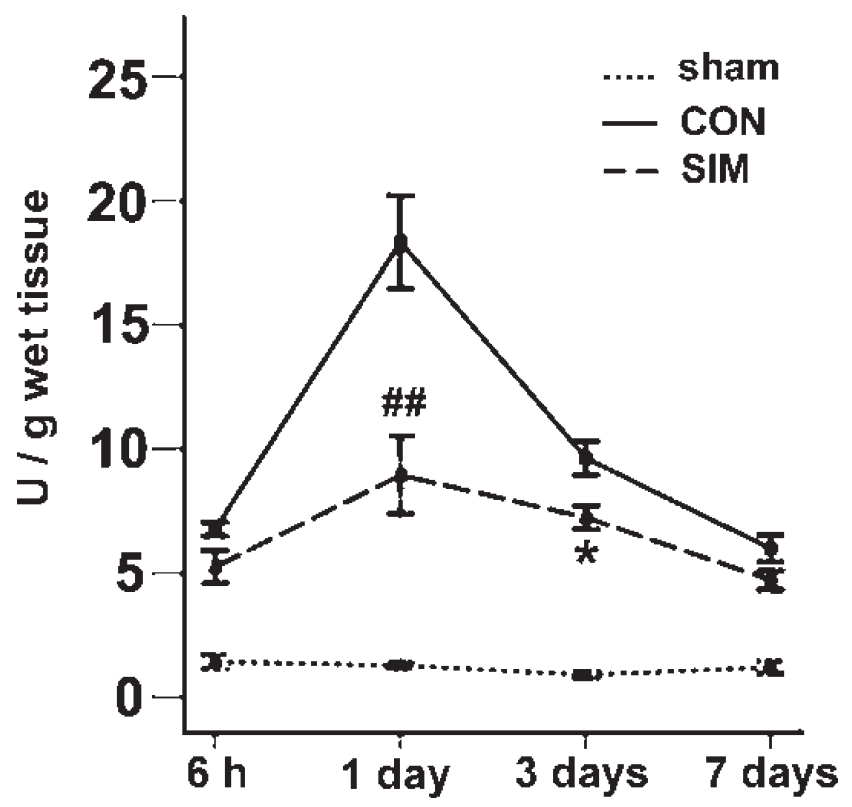

Figure 3. MPO activity in sham, CON, and SIM groups. MPO activity in the SIM group was significantly decreased at 1 day and 3 days after transplantation compared with the control group. Mean $\pm S E M, n=6$ rats per time point in each group. ${ }^{*} P<.05$, $\# \#<.01$ between CON and SIM groups.

demonstrated that pretreatment of atorvastatin for 1 or 3 days significantly reduced the infarct size, whereas pretreatment for 1 or 2 weeks failed. ${ }^{29}$ In this study, we planned to simulate the clinical settings where the exact moment of graft IR is usually indefinite; therefore, simvastatin administration was begun 3 days before operation and continued to the end point. In our current experimental settings, the perioperative treatment of simvastatin displayed a significant reduction of infarct size and myocardial fibrosis grade at 1 day after transplantation, consistent with the previous findings. ${ }^{15,22}$

Neutrophil and macrophage recruitment is a key feature of IRI. Gueler and colleagues ${ }^{30}$ revealed that short-term cerivastatin treatment significantly reduced ED1-positive macrophage infiltration in a rat renal IR model. However, in a mouse CAL model, Weinberg and colleagues ${ }^{13}$ failed to observe a marked change of neutrophil and CD45-positive leukocyte infiltration between the rosuvastatin-treated and control groups at 1 and 2 days after surgery. In agreement with these findings, our study showed that ED1-positive macrophage infiltration was also suppressed by simvastatin at 1 day and 3 days after transplantation. However, HIS4positive neutrophil accumulation was not obvious by simvastatin at 1 day after transplantation. Nevertheless, we still observed a significant reduction in the number of neutrophils 3 days after transplantation. Moreover, MPO activity, the quantitative index of neutrophil accumulation, has dis-

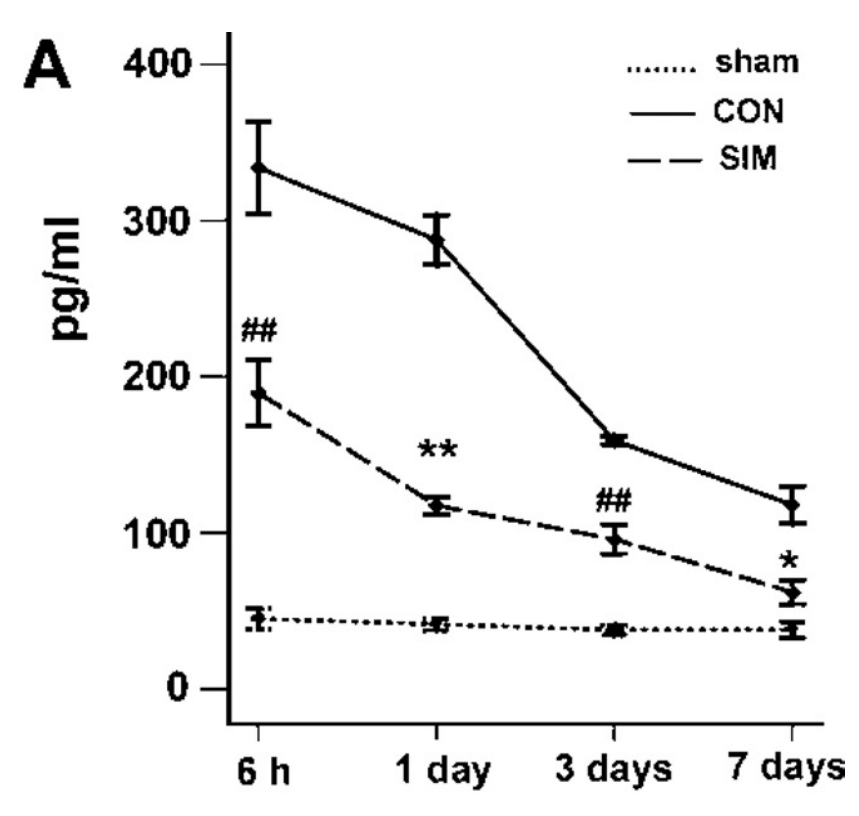

MCP-1 level in serum

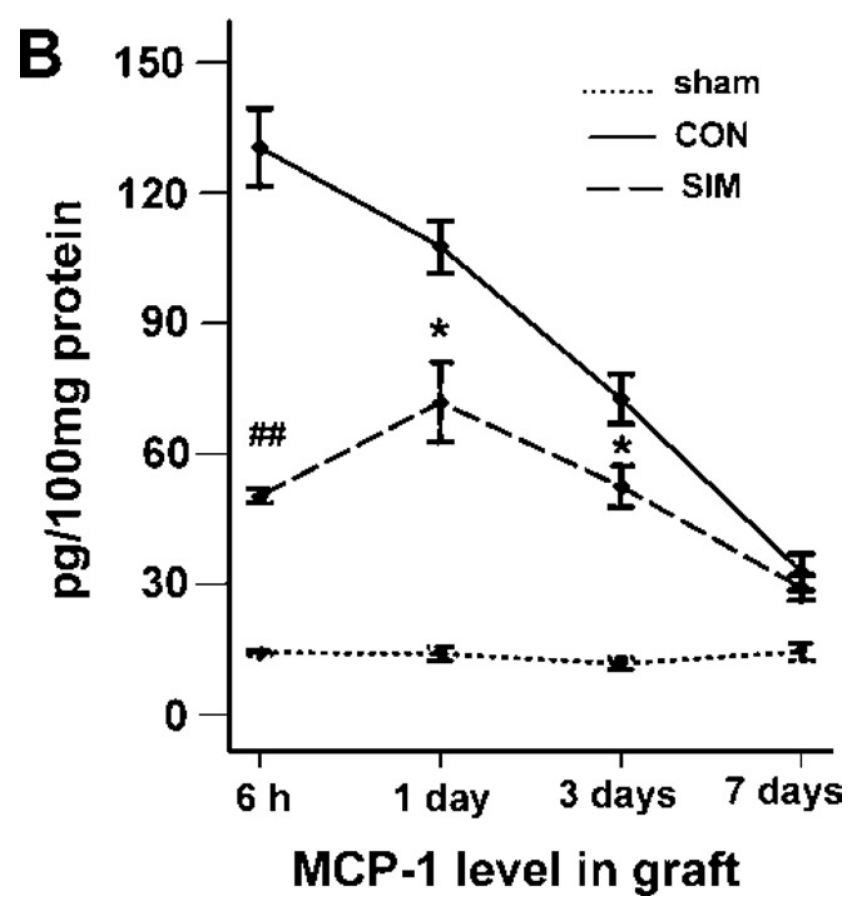

Figure 4. Concentration of MCP-1 in serum and isografts. Both MCP-1 levels in serum and grafts were significantly elevated in the CON group compared with the sham group. The serum level of MCP-1 was markedly decreased in the SIM group at each time point compared with the control group (A). The intragraft level of MCP-1 was not only decreased but also delayed in the SIM group compared with the control group (B). Mean $\pm S E M, \mathbf{n}=\mathbf{6}$ rats per time point in each group. ${ }^{*} P<.05, \# P<.01$, ${ }^{*} P<.001$ between CON and SIM groups. MCP-1, Monocyte chemoattractant protein-1. 


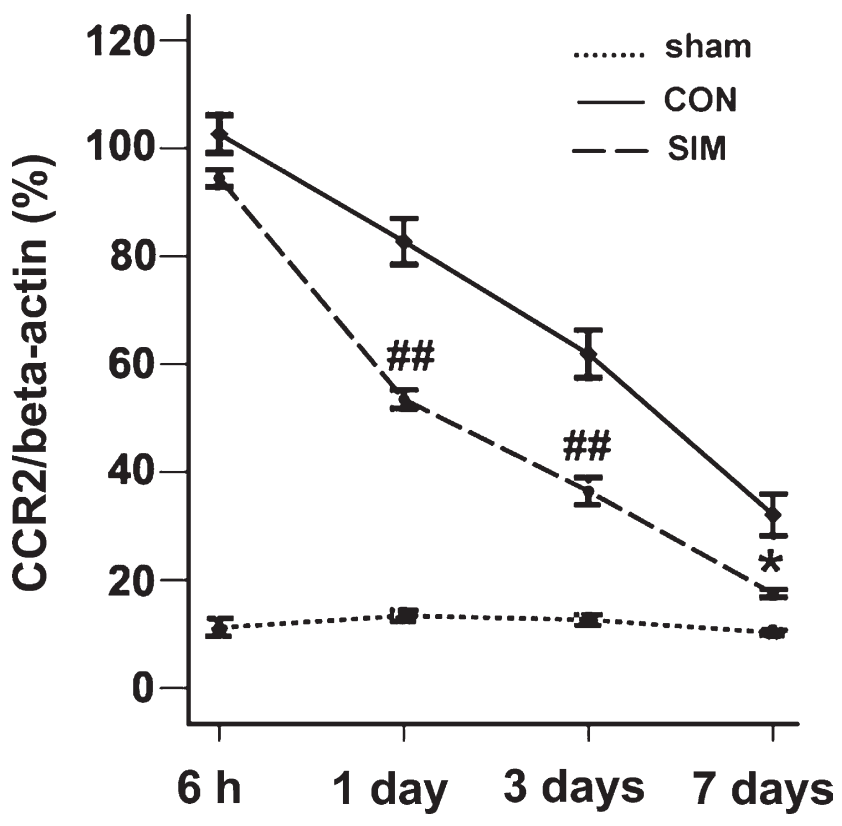

Figure 5. Quantitative real-time PCR of CCR2 mRNA expression in cardiac isografts. The expression of CCR2 was significantly upregulated in the CON group, which was reversed in the SIM group at 1,3 , and 7 days after transplantation. Mean $\pm S E M, n=6$ rats per time point in each group. ${ }^{*} P<.05, \# \#<.01$ between CON and SIM groups. CCR2, CC chemokine receptor-2.

played remarkable suppression at 1 day and 3 days after transplantation. Consequently, we believe that simvastatin may also alter neutrophil in addition to macrophage recruitment at the later stage of reperfusion.

MCP-1 induces monocyte migration in vitro and plays a critical role in mononuclear cells recruitment in vivo, which has been demonstrated. ${ }^{6}$ Tarzami and colleagues ${ }^{31}$ revealed that MCP-1 mRNA expression was increased after ischemia and peaked at 10 hours after ischemia, and that the MCP-1 protein level in hypoxic-treated myocytes was also markedly increased. Dewald and coworkers ${ }^{6,32}$ revealed that MCP-1 mRNA expression was rapidly induced at the early stage of myocardial IRI, peaked at 6 hours after reperfusion, and gradually decreased to baseline at 3 days after reperfusion in a mouse CAL model. Our study showed a similar time course of MCP-1 protein concentration in control serum and isograft tissues. In addition, accumulating evidence confirms that statins may suppress MCP-1 expression both in vitro and in vivo. However, most of the studies were performed in patients or animals with coronary artery disease $^{33-35}$ or in endothelial cells and macrophages stimulated by tumor necrosis factor- $\alpha$ or interferon- $\gamma .{ }^{16,36} \mathrm{Up}$ to the present, few researchers have considered the effect of statins on MCP-1 expression during IRI in transplantation, notwithstanding that IRI and inflammation usually coexist in the pathogenesis of coronary artery disease. In this study, we observed that MCP-1 levels in either serum or tissue were remarkably decreased in the SIM group compared with the control group. We found that simvastatin not only diminished the value of intragraft MCP-1 levels but also delayed its peaking to 1 day after transplantation. Indeed, suppression of macrophage infiltration should be the result of the significant reduction of MCP-1.

Finally, we analyzed the intragraft expression of CCR2, which has been studied by many investigators. The latest in vivo study ${ }^{37}$ demonstrated that CCR2 plays a critical role for firm adherence of leukocytes under postischemic conditions and the recruitment of leukocytes to reperfused tissue at later time points. Dewald and colleagues ${ }^{6}$ also reported that CCR2 showed a more prolonged induction peaking at 72 hours after reperfusion in untreated wild-type mice. However, our study found a different time course of CCR2 expression that peaked at 6 hours after transplantation, which was similar to the data reported by Tarzami and colleagues. ${ }^{31}$ Nevertheless, these results displayed a prolonged induction of CCR2 at the later phase of reperfusion. Accumulating evidence has indicated that the blockade of CCR2 signaling or the use of CCR2 knockout mice may alleviate left ventricular remodeling after myocardial infarction and myocardial or renal IRI. ${ }^{15,38}$ A recent study using 7ND-gene-transfection therapy significantly inhibited MCP-1/CCR2 signaling and retarded renal IRI. ${ }^{39}$ Consequently, target CCR2 may undoubtedly provide a new insight for myocardial IRI therapy. Moreover, a recent in vitro study ${ }^{16}$ indicated that simvastatin down-regulated the CCR2 expression of human endothelial cells and macrophages. The authors concluded that the inhibition of chemokine/chemokine receptors by simvastatin is GGPPdependent. The regulation of MCP-1 expression is related to the inhibition of statins on geranylgeranylated members of the Rho family guanosine triphosphatase. The activity of transcriptional repressor Oct-1 is also enhanced by statins and with a subsequent down-regulation of CCR2. In this study, simvastatin did not display significant downregulation of CCR2 at 6 hours after transplantation, although the mean value of the SIM group was lower than that of the control group. We believe that although CCR2 expression of recipient macrophages had been downregulated, the remnant leukocytes in the graft might upregulate CCR2 expression, which was insufficient to be alleviated with the dosage of simvastatin within 6 hours. However, we observed a remarkable reduction of CCR2 at the subsequent time points. Therefore, we considered that simvastatin retards the MCP- 1 secretion and CCR 2 expression of macrophages simultaneously by the GGPP pathway, which is followed by the reduction of macrophage infiltration and further decreases the intragraft concentration of MCP-1. Furthermore, a previous study 
using CCR2-deficient mice in a model of kidney IRI reduced the interstitial granulocyte infiltration. ${ }^{9}$ The authors explained that inflammation up-regulates CCR2 expression in granulocytes and that MCP-1 and CCR2 promote the chemotaxis of granulocytes during acute and chronic inflammatory conditions in vivo. Another independent investigation also indicated that secondary generation of proinflammatory mediators by activated $\mathrm{CCR} 2^{+}$monocytes is suggested to subsequently promote the recruitment of neutrophils. ${ }^{10}$ Consequently, down-regulation of CCR2 and MCP-1 by simvastatin may partially contribute to the suppression of both neutrophil and macrophage recruitment.

During the past 5 years, accumulating clinical studies have indicated that pretreatment of statins improves the postoperative prognosis and reduces the incidence of complications associated with cardiac surgery. ${ }^{40}$ Therefore, it is plausible for us to speculate that perioperative administration of statins initiated between 3 and 7 days before surgery may be beneficial for allograft survival. For example, the expected dosage of simvastatin may be approximately 40 to $80 \mathrm{mg}$ per day for each transplant recipient. Furthermore, despite our intriguing findings, several experimental limitations were noted for this study. First, the donor hearts of human beings should not be preserved in the simple way we described. Thus, heavy infarction could not emerge in the clinical settings, which may compromise the clinical relevance of this study. Second, we failed to address whether positive results are demonstrated with a longer ischemic duration in this study. Third, possible mechanisms involved were not exclusively investigated, such as the antioxidant effects and the ability of up-regulating endothelial nitric oxide synthase expression. Further study is warranted to better elucidate the signaling mechanisms involved and dose-effectiveness relationship for simvastatin.

\section{Conclusions}

Our experiment demonstrated for the first time that perioperative treatment of simvastatin suppressed myocardial IRI independently of cholesterol-lowering effects in a rat model of cardiac isograft transplantation. Our data revealed that attenuation of neutrophil and macrophage infiltration by inhibiting CCR2 expression and reducing MCP-1 level may serve as a possible mechanism of action for the protective effects elicited by simvastatin. On the basis of these findings, we speculated that perioperative treatment of statins initiated at 3 to 7 days before surgery may be beneficial for heart allograft survival in the clinical setting.

The authors appreciate the technical assistance from Drs Genbao Feng, Qui Meng, and Hao Ma.

\section{References}

1. Anaya-Prado R, Toledo-Pereyra LH, Lentsch AB, Ward PA. Ischemia/ reperfusion injury. J Surg Res. 2002;105:248-58.
2. Hirayama S, Shiraishi T, Shirakusa T, Higuchi T, Miller EJ. Prevention of neutrophil migration ameliorates rat lung allograft rejection. Mol Med. 2006;12:208-13.

3. Tanaka M, Mokhtari GK, Terry RD, Gunawan F, Balsam LB, Hoyt G, et al. Prolonged cold ischemia in rat cardiac allografts promotes ischemia-reperfusion injury and the development of graft coronary artery disease in a linear fashion. $J$ Heart Lung Transplant. 2005;24: 1906-14.

4. Yamani MH, Haji SA, Starling RC, Tuzcu EM, Ratliff NB, Cook DJ, et al. Myocardial ischemic-fibrotic injury after human heart transplantation is associated with increased progression of vasculopathy, decreased cellular rejection and poor long-term outcome. J Am Coll Cardiol. 2002;39:970-7.

5. Frangogiannis NG, Entman ML. Targeting the chemokines in myocardial inflammation. Circulation. 2004;110:1341-2.

6. Dewald O, Zymek P, Winkelmann K, Koerting A, Ren G, AbouKhamis T, et al. CCL2/monocyte chemoattractant protein-1 regulates inflammatory responses critical to healing myocardial infarcts. Circ Res. 2005;96:881-9.

7. Frangogiannis NG, Entman ML. Chemokines in myocardial ischemia. Trends Cardiovasc Med. 2005;15:163-9.

8. Hayasaki T, Kaikita K, Okuma T, Yamamoto E, Kuziel WA, Ogawa $\mathrm{H}$, et al. CC chemokine receptor-2 deficiency attenuates oxidative stress and infarct size caused by myocardial ischemia-reperfusion in mice. Circ J. 2006;70:342-51.

9. Furuichi K, Wada T, Iwata Y, Kitagawa K, Kobayashi K, Hashimoto $\mathrm{H}$, et al. CCR2 signaling contributes to ischemia-reperfusion injury in kidney. J Am Soc Nephrol. 2003;14:2503-15.

10. Maus UA, Waelsch K, Kuziel WA, Delbeck T, Mack M, Blackwell TS, et al. Monocytes are potent facilitators of alveolar neutrophil emigration during lung inflammation: role of the CCL2-CCR2 axis. J Immunol. 2003;170:3273-8.

11. Undas A, Celinska-Lowenhoff M, Kaczor M, Musial J. New nonlipid effects of statins and their clinical relevance in cardiovascular disease. Thromb Haemost. 2004;91:1065-77.

12. Davignon J. Beneficial cardiovascular pleiotropic effects of statins. Circulation. 2004;109(23 Suppl 1):III39-43.

13. Weinberg EO, Scherrer-Crosbie M, Picard MH, Nasseri BA, MacGillivray C, Gannon J, et al. Rosuvastatin reduces experimental left ventricular infarct size after ischemia-reperfusion injury but not total coronary occlusion. Am J Physiol Heart Circ Physiol. 2005;288: H1802-9.

14. Wolfrum S, Dendorfer A, Schutt M, Weidtmann B, Heep A, Tempel $\mathrm{K}$, et al. Simvastatin acutely reduces myocardial reperfusion injury in vivo by activating the phosphatidylinositide 3-kinase/Akt pathway. J Cardiovasc Pharmacol. 2004;44:348-55.

15. Efthymiou CA, Mocanu MM, Yellon DM. Atorvastatin and myocardial reperfusion injury: new pleiotropic effect implicating multiple prosurvival signaling. J Cardiovasc Pharmacol. 2005;45:247-52.

16. Veillard NR, Braunersreuther V, Arnaud C, Burger F, Pelli G, Steffens $\mathrm{S}$, et al. Simvastatin modulates chemokine and chemokine receptor expression by geranylgeranyl isoprenoid pathway in human endothelial cells and macrophages. Atherosclerosis. 2006;188:51-8.

17. Scalia R, Gooszen ME, Jones SP, Hoffmeyer M, Rimmer DM 3rd, Trocha SD, et al. Simvastatin exerts both anti-inflammatory and cardioprotective effects in apolipoprotein E-deficient mice. Circulation. 2001;103:2598-603.

18. Ono K, Lindsey ES. Improved technique of heart transplantation in rats. J Thorac Cardiovasc Surg. 1969;57:225-9.

19. Akashi S, Sho M, Kashizuka H, Hamada K, Ikeda N, Kuzumoto Y, et al. A novel small-molecule compound targeting CCR5 and CXCR3 prevents acute and chronic allograft rejection. Transplantation. 2005; 80:378-84.

20. Naidu BV, Woolley SM, Farivar AS, Thomas R, Fraga C, Mulligan MS. Simvastatin ameliorates injury in an experimental model of lung ischemia-reperfusion. J Thorac Cardiovasc Surg. 2003;126:482-9.

21. Landmesser U, Engberding N, Bahlmann FH, Schaefer A, Wiencke A, Heineke A, et al. Statin-induced improvement of endothelial progenitor cell mobilization, myocardial neovascularization, left ventricular function, and survival after experimental myocardial infarction requires endothelial nitric oxide synthase. Circulation. 2004;110:1933-9. 
22. Bell RM, Yellon DM. Atorvastatin, administered at the onset of reperfusion, and independent of lipid lowering, protects the myocardium by up-regulating a pro-survival pathway. J Am Coll Cardiol. 2003;41:508-15

23. Lazar HL, Bao Y, Zhang Y, Bernard SA. Pretreatment with statins enhances myocardial protection during coronary revascularization. J Thorac Cardiovasc Surg. 2003;125:1037-42.

24. Tiefenbacher CP, Kapitza J, Dietz V, Lee CH, Niroomand F. Reduction of myocardial infarct size by fluvastatin. Am J Physiol Heart Circ Physiol. 2003;285:H59-64.

25. Di Napoli P, Taccardi AA, Grilli A, De Lutiis MA, Barsotti A, Felaco $\mathrm{M}$, et al. Chronic treatment with rosuvastatin modulates nitric oxide synthase expression and reduces ischemia-reperfusion injury in rat hearts. Cardiovasc Res. 2005;66:462-71.

26. Lefer AM, Campbell B, Shin YK, Scalia R, Hayward R, Lefer DJ. Simvastatin preserves the ischemic-reperfused myocardium in normocholesterolemic rat hearts. Circulation. 1999;100:178-84.

27. Sutherland FJ, Hearse DJ. The isolated blood and perfusion fluid perfused heart. Pharmacol Res. 2000;41:613-27.

28. Jones SP, Trocha SD, Lefer DJ. Pretreatment with simvastatin attenuates myocardial dysfunction after ischemia and chronic reperfusion. Arterioscler Thromb Vasc Biol. 2001;21:2059-64.

29. Mensah K, Mocanu MM, Yellon DM. Failure to protect the myocardium against ischemia/reperfusion injury after chronic atorvastatin treatment is recaptured by acute atorvastatin treatment: a potential role for phosphatase and tensin homolog deleted on chromosome ten? J Am Coll Cardiol. 2005;45:1287-91.

30. Gueler F, Rong S, Park JK, Fiebeler A, Menne J, Elger M, et al. Postischemic acute renal failure is reduced by short-term statin treatment in a rat model. J Am Soc Nephrol. 2002;13:2288-98.

31. Tarzami ST, Cheng R, Miao W, Kitsis RN, Berman JW. Chemokine expression in myocardial ischemia: MIP-2 dependent MCP-1 expression protects cardiomyocytes from cell death. J Mol Cell Cardiol. 2002;34:209-21.
32. Dewald O, Ren G, Duerr GD, Zoerlein M, Klemm C, Gersch C, et al. Of mice and dogs: species-specific differences in the inflammatory response following myocardial infarction. Am J Pathol. 2004;164: 665-77.

33. Rezaie-Majd A, Maca T, Bucek RA, Valent P, Muller MR, Husslein P, et al. Simvastatin reduces expression of cytokines interleukin-6, interleukin-8, and monocyte chemoattractant protein-1 in circulating monocytes from hypercholesterolemic patients. Arterioscler Thromb Vasc Biol. 2002;22:1194-9.

34. Bustos C, Hernandez-Presa MA, Ortego M, Tunon J, Ortega L, Perez $\mathrm{F}$, et al. HMG-CoA reductase inhibition by atorvastatin reduces neointimal inflammation in a rabbit model of atherosclerosis. J Am Coll Cardiol. 1998;32:2057-64.

35. Xu ZM, Zhao SP, Li QZ, Nie S, Zhou HN. Atorvastatin reduces plasma MCP-1 in patients with acute coronary syndrome. Clin Chim Acta. 2003;338:17-24.

36. Ortego M, Bustos C, Hernandez-Presa MA, Tunon J, Diaz C, Hernandez G, et al. Atorvastatin reduces NF-kappaB activation and chemokine expression in vascular smooth muscle cells and mononuclear cells. Atherosclerosis. 1999;147:253-61.

37. Reichel CA, Khandoga A, Anders HJ, Schlondorff D, Luckow B, Krombach F. Chemokine receptors Ccr1, Ccr2, and Ccr5 mediate neutrophil migration to postischemic tissue. J Leukoc Biol. 2006;79: 114-22.

38. Kaikita K, Hayasaki T, Okuma T, Kuziel WA, Ogawa H, Takeya M. Targeted deletion of CC chemokine receptor 2 attenuates left ventricular remodeling after experimental myocardial infarction. Am J Pathol. 2004; 165:439-47.

39. Furuichi K, Wada T, Iwata Y, Kitagawa K, Kobayashi K, Hashimoto $\mathrm{H}$, et al. Gene therapy expressing amino-terminal truncated monocyte chemoattractant protein-1 prevents renal ischemia-reperfusion injury. J Am Soc Nephrol. 2003;14:1066-71.

40. Hindler K, Shaw AD, Samuels J, et al. Improved postoperative outcomes associated with preoperative statin therapy. Anesthesiology. 2006;105:1260-72; quiz 1289-90. 